\title{
Schulddogmatik in der bundesgerichtlichen Rechtsprechung
}

Zugleich Besprechung des Urteils des Bundesgerichts 6B_1363/2019 vom 19. November 2020

Mit seinem Urteil 6B_1363/2019 hat sich das Bundesgericht zu einer Fülle von dogmatischen Streitfragen im Schuldkontext geäussert. Es bekannte sich grundsätzlich zu einem sozialen Schuldbegriff. Die Autoren legen dar, dass ein funktionaler Schuldbegriff in seinem theoretischen Fundament überzeugender ist. Auf Basis dieser Erkenntnis wird aufgezeigt, dass sich die Auffassung des Bundesgerichts, bei schweren Straftaten hohe Anforderungen an eine vollständige Aufhebung der Schuldfähigkeit zu stellen, in ihrer Generalisierung selbst bei einem funktionalen Schuldverständnis nicht halten lässt. Schliesslich wird auch der bundesgerichtlichen Auffassung widersprochen, wonach sich die forensisch-psychiatrisch sachverständige Person zur Frage der Schuldfähigkeit äussern dürfe.

I. Einleitung

II. Der materielle Schuldbegriff des Bundesgerichts: sozial oder funktional?

III. Schuldmassstab bei schweren Straftaten (implizite Hemmschwellentheorie)

IV. Zulässigkeit von gutachterlichen Empfehlungen zur Schuldfähigkeit

Zitiervorschlag:

THIERRY URWYLER / LUCA RANZONI, Schulddogmatik in der bundesgerichtlichen Rechtsprechung, sui generis 2021, S. 179

Dr. iur. Thierry Urwyler, Lehrbeauftragter an der Universität Luzern und der Universität Zürich (thierry urwyler@hotmail.com).

Luca Ranzoni, RA MLaw, LL.M. (Maastricht), Assistent und Doktorand am Lehrstuhl für Straf- und Strafprozessrecht von Prof. Dr. iur. Marc Thommen an der Universität Zürich (lucaranzoni@msn.com). Die Autoren bedanken sich bei Prof. Dr. Alexander Baur, Dr. Gian Ege, MLaw Franziska Rader und Prof. Dr. Monika Simmler für die kritische Durchsicht des Manuskripts und die wertvollen Anmerkungen.

URL: sui-generis.ch/181

DOI: https://doi.org/10.21257/sg.181

Dieses Werk ist lizenziert unter einer Creative Commons Namensnennung - Weitergabe unter gleichen Bedingungen 4.0 International Lizenz. 


\section{Einleitung}

1 Grundlage des hier zu referierenden Urteils bildet ein tragischer Sachverhalt: Ein IT-Doktorand hat stark alkoholisiert einen Lehrling morgens um 4 Uhr an der Tramhaltestelle Milchbuck (Zürich) mit massiver Gewalt attackiert und schwer verletzt. In der darauffolgenden Strafuntersuchung mit dem Vorwurf der (eventual-)vorsätzlichen Tötung spielte die Schuldfähigkeit des Beschuldigten eine zentrale Rolle. Der aus diesem Rechtsstreit entstandene Entscheid 6B_1363/20191 darf in vielerlei Hinsicht als Sonderfall eingestuft werden. Den Autoren ist kein anderes Urteil bekannt, in welchem sich das Bundesgericht zu so vielen dogmatischen Streitfragen im Schuldkontext in vergleichbarer Tiefe geäussert hat. Aufgrund der Breite der aufgeworfenen Rechtsfragen wollen sich die Autoren auf drei Aspekte beschränken: In einem ersten Schritt soll der Frage nachgegangen werden, welchen materiellen Schuldbegriff das Bundesgericht vertritt (II.). Danach soll die Thematik der erhöhten Anforderungen für verminderte oder aufgehobene Schuldfähigkeit bei Schwerstdelikten durchleuchtet werden (III.). Zuletzt ist der Frage nachzugehen, inwiefern es erlaubt ist, dass sich die sachverständige Person zur Rechtsfrage der Schuldfähigkeit äussert (IV.).

\section{Der materielle Schuldbegriff des Bundesgerichts: sozial oder funktional?}

2 Bei der Prüfung des Schuldvorwurfs stellte das Bundesgericht Folgendes fest: Ob gestützt auf die gutachterlichen Erkenntnisse von verminderter Schuldfähigkeit oder Schuldunfähigkeit auszugehen sei, enthalte eine normative Komponente. Positiv feststellen lasse sich nur die Einsichtsfähigkeit. Die Steuerungsfähigkeit bzw. generelle Existenz einer Willensfreiheit lasse sich hingegen nicht mit empirischen Mitteln messen. Die Steuerungsfähigkeit positiv feststellen zu wollen, laufe auf den Nachweis der Willensfreiheit hinaus, den es nicht geben könne. Die Lehre anerkenne, dass es sich bei der Beurteilung der Steuerungsfähigkeit um eine normative $\mathrm{Zu}$ schreibung handle, «welchenicht auf einem individuellen ‘Anders-Handeln-Können beruht, sondern sich an der Leitlinie einer «massgeschneiderten` Durchschnittsperson in der gleichen Situation wie der Täter orientiert, welcher nach der Erfahrung Handlungsspielräume zur Verfügung gestanden hätten». Es bestehe erheblicher Spielraum für normative Entscheidungen. In die gleiche Richtung gehe der in der Lehre verwendete soziale Schuldbegriff, der anerkenne, dass im Schuldmassstab ein gewisses Mass an

1 Urteil des Bundesgerichts 6B 1363/2019 vom 19. November 2020 E.1.6.2.
Generalisierung stecke, «da die Fähigkeit des Täters, sich in der Tatsituation normgemäss zu verhalten, von ihm in bestimmten Grenzen kurzerhand gefordert, das heisst als vorhanden vorausgesetzt wird». Der soziale Schuldbegriff dürfe das Schuldstrafrecht aber nicht in Frage stellen. ${ }^{2}$

So plausibel die bundesgerichtlichen Erwägungen klin- 3 gen, so sehr verdecken sie die dogmatische Komplexität der zugrunde liegenden Fragen. Sobald man von einem Alltagsverständnis in die rechtswissenschaftliche Diskussion abtaucht, ist nicht mehr evident, was unter Schuld inhaltlich zu verstehen ist (materieller Schuldbegriff). ${ }^{3}$

In seinem Urteil stellt das Bundesgericht sein Schuldver- 4 ständnis in die Nähe des «sozialen» Schuldbegriffs. ${ }^{4} \mathrm{Da}-$ mit wird einem herkömmlichen ${ }^{5}$ (auch: traditionellen) Schuldverständnis, welches Schuld als Möglichkeit des realen Anders-Handeln-Könnens der individuellen Täterin versteht ${ }^{6}$, eine klare und berechtigte Absage erteilt. ${ }^{7}$ Ein solcher individuell-sittlicher Vorwurf an die Täterin kann nicht aufrechterhalten werden, da - wie vom Bundesgericht zurecht betont - der Nachweis einer Willensfreiheit (ungeachtet der neurobiologischen Forschungsergebnisse ${ }^{8}$ ) nicht gelingen kann und entsprechend (in dubio pro reo) immer ein Freispruch erfolgen müsste. ${ }^{9}$ Dieses Problem ist seit langer Zeit bekannt und hat in der schweizerischen Lehre und Rechtsprechung zu Adaptionen im Sinne eines sozialen Schuldbegriffs geführt: Im Schuldmassstab stecke «ein beträchtliches Quantum an

2 Für dengesamten Abschnitt: Urteil des Bundesgerichts 6B_1363/2019 vom 19. November 2020 E.1.6.2.

3 Zu den zahlreichen unterschiedlichen Konzeptionen ausführlich CLAUS ROXIN / LUIS GRECO, Strafrecht Allgemeiner Teil, Bd.1: Grundlagen. Der Aufbau der Verbrechenslehre, 5. Aufl., München $2020, \$ 19$.

4 Dabei lässt es unglücklicherweise offen, ob es sein Verständnis letztlich daran ausrichtet oder ob der bundesgerichtliche materielle Schuldbegriff doch noch durch «eigene» Merkmale charakterisiert ist, die im sozialen Schuldbegriff der Lehre nicht enthalten sind.

5 Zum Begriff: DANIEL SCHMID, Krank oder böse?, Die Schuldfähigkeit und die Sanktionenindikation dissozial persönlichkeitsgestörter Straftäter und delinquenter «Psychopaths» sowie die Zusammenarbeit von Jurisprudenz und Psychiatrie bei der Beurteilung der Schuldfähigkeit, Basel 2009, S. 158

6 So etwa vom Bundesgerichtshof in Deutschland vertreten: Urteil des Bundesgerichtshofs GSSt 2/51 vom 18. März 1952, Rz.15(BGHSt 2, 194); So wohl auch Teile der früheren Rechtsprechung des Bundesgerichts. Vgl. BGE 80 IV 234 E. 2 («mit dem vorhandenen Teil von Willensfreiheit, für den er einzustehen hat»). Mit «vorhanden» wird angedeutet, dass das Bundesgericht die Willensfreiheit als etwas Vorhandenes, empirisch Fassbares erachtet. Ähnlich in BGE 85 IV 95, wo das Bundesgericht die Steuerungsfähigkeit als psychische Eigenschaft bezeichnet.

7 Zur Kritik am traditionellen Schuldbegriff auch MONIKA SIMMLER, Normstabilisierung und Schuldvorwurf, Diss. Zürich, Bern 2018, S. $425 \mathrm{ff}$.

8 Dazu GIAN EGE, Das Strafrecht und die Hirnforschung, ZStrR 135/2017, S. $299 \mathrm{ff}$.

9 ROXIN/GRECO (Fn.3), §19 N21. 
Generalisierung» ${ }^{10} \mathrm{bzw}$. der Schuldvorwurfberuhe nicht auf einem individuellen, sondern einem «generellen Anders-Handeln-Können» (Massstab: Durchschnittsperson in der Situation der Täterin) ${ }^{\mathbf{1 1}}$. Dies sei nötig, da «dievolle Individualisierung des Schuldmassstabes zu sehr weitgehender Exkulpation des Täters führen und damit schliesslich das Schuldprinzip selbst in Frage stellen könnte.»12

5 Das Problem eines mit solchen Generalisierungen arbeitenden (sozialen ${ }^{13}$ ) Schuldverständnisses wird jedoch von ROXIN/GRECO auf den Punkt gebracht: Einen individuellen sittlichen Vorwurf gegen eine konkrete Person kann man «unmöglich aufFähigkeiten stützen, die andere Personen vielleicht haben, die aber dem Täter gerade fehlen! Das ist nicht nur unlogisch, sondern auch eine Preisgabe des Ausgangspunktes, dass dem Täter selbst eine freie Entscheidung möglich sein muss.»14 Ist der Rechtsstaat verpflichtet, die Voraussetzungen von Grundrechtseingriffen einer rationalen Überprüfung zugänglich zu machen, gelingt dies nicht, wenn der Anknüpfungssachverhalt eines individuellen Vorwurfs (Willensfreiheit) dem Beweis nicht zugänglich ist. ${ }^{15}$ Diesem Problem kann man auch nicht entgehen, indem man - wie ein Teil der Lehre - den materiellen Schuldbegriff als eine metaphysische und auf Gerechtigkeit zielende Einheit definiert, die nicht durch empirische Mittel messbar ist ${ }^{16}$ : In einem Rechtsstaat sollten Grundrechtseingriffe wie die Strafe

10 GÜNTER STRATENWERTH, Schweizerisches Strafrecht, Allgemeiner Teil I: Die Straftat, 4. Aufl., Bern 2011, §11 N6.

11 FELIX BOMMER, in: Niggli/Wiprächtiger(Hrsg.), Basler Kommentar, Strafrecht, 4. Aufl., Basel 2018, Vor Art. 19 N18 (zit. BSK StGB-BEARBEITERIN).

12 STRATENWERTH, ATI(Fn.10), \$11 N6; diese Problematik wird in der Literatur unter dem Stichwort «Dammbruchgefahr» geführt. Vgl. FRANZ STRENG, Schuld ohne Freiheit? Der funktionale Schuldbegriff auf dem Prüfstand, ZStW 1989, S. 277.

13 Wobei man sich als juristischer Laie fragen könnte, was an einem solchen Verständnis sozial sei, wenn damit einem Individuum Fähigkeiten zugeschrieben werden, die dieses Individuum schlicht nicht hat. Für einen Laien wäre ein solcher Schuldbegriff wohl eher unsozial (zum Begriff: Duden, unsozial).

14 ROXIN/GRECO (Fn.3), §19 N22; vgl. auch PETER ALBRECHT, Strafzumessung ohne individuelle Tatschuld?, ZStrR 2017, S. 454, der darüber ein «schleichendes Unbehagen» verspürt, da Beschuldigte im Strafverfahren für die ihnen vorgeworfenen Delikte «schuldig» erklärt werden, «obwohl eine persönliche Verantwortung im Sinne eines Anders-handeln-Könnens forensisch nicht nachweisbar ist»; ebenso STRENG, Schuld ohne Freiheit? (Fn.12), S. 279 «Mit diesem -scheinbaren - Ausweg aus dem Problem der Unbeweisbarkeit individueller Handlungsfreiheit verliert man aber jede Legitimation für einen auf Freiheitsmißbrauch gestützten individualethischen Tadel gegen diesen Täter».

15 Dies anerkennt auch BOMMER, wenn er schreibt, dass «sich eine $a b$ solute Straftheorie in der dargestellten ‘absoluten〉 Form nicht halten lässt. Gegen siesprechen alle Einwände, die sich gegen die Feststellung (wirklicher) Schuld richten, insb. die Unentscheidbarkeit der Frage nach der Willensfreiheit», vgl. BSK StGB-BOMMER, Vor Art.19 N 47.

16 So MARCEL A. NIGGLI / STEFAN MAEDER, Die funktionale Stellung der Schuld in absoluten und relativen Straftheorien, in: Queloz/ Niggli/Riedo (Hrsg.), Droit pénal et diversités culturelles, FSJosé Hurtado Pozo, Genf 2012, S. 316. nicht durch Metaphysik, sondern das unserer Wahrnehmung Zugängliche (und somit Falsifizierbare) legitimiert werden. ${ }^{17}$

Als Reaktion auf die wahrgenommenen Defizite des tra- 6 ditionellen und des sozialen Schuldbegriffs hat die Lehre andere Konzeptionen des materiellen Schuldbegriffs vorgeschlagen. Es besteht kein Raum, sämtliche Ansätze nachzuzeichnen. ${ }^{18}$ Beachtung verdient indes der sog. funktionale Schuldbegriff, wie er in Deutschland z.B. von STRENG und JAKOBS und in der Schweiz von SIMMLER vertreten wird (je nach Autor/in mit leicht abweichender Akzentsetzung). ${ }^{19}$ Sehr verdichtet ausgedrückt ist der Ausgangspunkt eines funktionalen Schuldbegriffs die Beobachtung, dass Menschen subjektiv Freiheit empfinden (dieses Gefühl besteht - und zwar selbst dann, wenn wir faktisch vollständig determiniert wären) ${ }^{20}$, und dass Menschen sich auf diesem Gefühl basierend Verantwortung zuschreiben, was sich (und dies ist die Funktion) positiv auf den Erhalt der Gesellschaftsordnung bzw. das gesellschaftliche Zusammenleben auswirkt (Normstabilisierung). Solche Schuldverständnisse knüpfen zwar an die empirisch fassbaren Korrelate der Steuerungsfähigkeit ${ }^{21}$ (bzw. jene, welche Menschen damit in Verbindung bringen) an. Dies erfolgt aber im Bewusstsein, dass man mit diesem Schuldverständnis nicht an eine objektive Willensfreiheit anknüpft, sondern das, was Menschen nach ihrem subjektiven Freiheitsempfinden mit ihr in Verbindung bringen. Daher ist der Inhalt der so formulierten materiellen Schuldbegriffe nicht, wie landläufig vermutet (und wie im traditionellen Schuldbegriff enthalten), ein «Du hättest faktisch anders handeln können und weil Du es nicht getan hast, machen wir dir einen individuell-ethischen Vorwurf», sondern nach Sicht der Autoren ein «Wir als Gesellschaft sind auf Basis unserer Freiheitsgefühls-Erfahrung der Auffassung, dass Dir beider Tat

17 Vgl. auch STRENG, Schuld ohne Freiheit? (Fn. 12), S. 280: «[...] die Verwirklichung metaphysischer Postulate kann in einer modernen Demokratie keine Staatsaufgabe sein".

18 Eine Übersicht findet sich bei ROXIN/GRECO (Fn.3), \$19 N1ff.

19 GÜNTHER JAKoBS, Strafrecht, Allgemeiner Teil, Die Grundlagen und die Zurechnungslehre. Berlin 1991, S. 476 ff.; gem. STRENG, Schuld ohne Freiheit? (Fn.12), handelt es sich beim funktionalen Schuldbegriff um eine «spezielle Variante des sozialen Schuldverständnisses», S. 275 und dort Fn. 10 in fine; für eine vertiefte Auseinandersetzung mit STRENGS und JAKOBS Konzeptionen siehe bei ANGELA KALOUS, Positive Generalprävention durch Vergeltung, Regensburg 2000, S. 87 ff. und $130 \mathrm{ff}$; f ferner SIMMLER (Fn.7), S. $1 \mathrm{ff}$.

20 STRENG, Schuld ohne Freiheit? (Fn.12), S. 298.

21 Gemeint ist mit diesen empirischen Korrelaten das «allgegenwärtige Erleben eigener Freiheitsempfindungen», so STRENG, Schuld ohne Freiheit? (Fn.12), S. 298. Vgl. weiter den ähnlichen Gedanken bei ROXIN/GRECO (Fn.3), \$19 N 46: «Die Schuld ist demnach bei der hier favorisierten Auffassung eine gemischt empirisch-normative Gegebenheit. Empirisch feststellbar ist die prinzipielle Fähigkeit zur Selbststeuerung und die damit gegebene normative Ansprechbarkeit; (damit wird nicht geleugnet, dass im Grenzbereich dieser Ansprechbarkeit Wertungen einfließen, doch ist dies bei allen Rechtsbegriffen so).» 
Freiheitsräumefür Handlungsalternativen zur Verfügung standen. Weildu diesenichtgenutzt hast, schreiben wir Dir die individuelle Verantwortung zu und auferlegen Dir die Schuldstrafe, da wir das als gerecht empfinden und diesfür den Erhalt der Rechtsordnung notwendig ist».

7 Dieser Formulierungsunterschied ist von zentraler Bedeutung: Nicht die «reale» Willensfreiheit, sondern unser subjektives Freiheitsempfinden ist Grundlage der wechselseitigen Verantwortungszuschreibung zwischen Menschen $^{22}$ und dies wiederum ist eine wichtige Bedingung eines geordneten Zusammenlebens. ${ }^{23}$ Von diesem Prinzip (Verantwortungszuschreibung) sieht die Gesellschaft nur ab, wenn die von Menschen empfundenen Freiheitsräume in den Dimensionen des Erkennens und Steuerns bei einer Person im Tatzeitpunkt störungsbedingt ausgeschaltet oder zumindest erheblich beeinträchtigt waren. Gerade mit dieser Beschreibung tragen die Theorien rund um funktionale Schuldverständnisse zur Versachlichung bei: «Das große Verdienst desfunktionalen Schuldbegriffs, das ihm aber selten angerechnet wird, ist der in ihm verkörperte Beitrag zur Selbsternüchterung bzw. zur Aufrichtigkeit in der Diskussion über die strafrechtliche Schuld. Denn hinter der gelegentlich sogar emphatischen Zurückweisung der Theorie steht häufig nichts anderes als die Sache: Nicht selten dient die verbreitete individualistische Willensfreiheitsrhetorik gerade der Kaschierung der in Wahrheitmaßgeblichen sozialen Präventionsbelange.»24

8 Weshalb hat sich nun ein wesentlicher Teil des Schrifttums für einen sozialen (z.T. auch "pragmatisch-sozialen») und nicht für einen funktionalen Schuldbegriff ausgesprochen? Massgeblich dürften die vorliegenden Einwände sein: (i) Schuld sei im funktionalen Schuldbegriff ein reines Etikett, mit dem (ii) die betroffene Person zum Instrument für Normbestätigungsbedürfnisse missbraucht werde, was mit der Menschenwürde (Art.7 BV) unvereinbar sei. ${ }^{25}$ Weiter verliere man (iii) mit diesem Schuldverständnis den Vorteil der strafbarkeitsbegren-

22 Vgl. auch SIMMLER (Fn. 7), S. 427, die in diesem Zusammenhang die berechtigte Frage aufwirft, ob man den beladenen Schuldbegriff nicht verabschieden und schlicht von Verantwortungszuschreibung sprechen sollte. Vgl. auch ALBRECHT (Fn.14), S. 453: «Im Zusammenleben mit anderen Personen besteht offenkundig ein Verlangen nach Zurechnung bzw. Zuschreibung von Verantwortung.»

23 So auch SIMMLER (Fn. 7), S. 427: «So ist es nicht das Andershandelnkönnen selbst, welches die Strafe rechtfertigt, sondern vielmehr die funktionale Notwendigkeit der Verantwortungszuschreibung für die strafrechtliche Normstabilisierung und damit für den Erhalt der sozialen Ordnung.»

24 ROXIN/GRECO (Fn.3), \$19 N35b.

$25 \mathrm{Zu}$ (i): ROXIN/GRECO (Fn. 3), §19 N 52h; vgl. auch die Nachweise bei TOBIAS R. ANDRISSEK, Vergeltung als Strafzweck, Empirisch-soziologische Begründung und kriminalpolitische Folgerungen, Tübingen 2017, S. 124; zu (ii): ausführlich FRANK NEUSS, Der Strafzweck der Generalprävention im Verhältnis zur Würde des Menschen, Aachen 2001, insb. S.151ff.; MATTHIAS MÜLLER, Vergeltungsstrafe und Gerechtigkeitsforschung, Tübingen 2019, S.144ff. zenden Wirkung des Schuldprinzips (Garantie- und Schutzfunktion des Schuldprinzips $\left.{ }^{26}\right) .{ }^{27}$ Schliesslich lasse sich (iv) die positiv-generalpräventive Prämisse der Normstabilisierungswirkung durch Strafe nicht empirisch überprüfen. ${ }^{28}$

Angesichts dieser schwerwiegenden Einwände könnte 9 eine Versuchung darin bestehen, sich reflexartig zurückzuziehen und das Glück im sozialen Schuldbegriff zu suchen. Dies wäre aufgrund der angesprochenen Schwächen dieses Konzepts aber kaum überzeugend (hiervor Rz. 5). Konsequenter ist es darum, die Einwände gegen den funktionalen Schuldbegriff genauer zu beleuchten und sie im Gesamtkontext mit dem sozialen Schuldbegriff zu betrachten.

Bei dieser Analyse fällt erstens auf, dass die Einwände 10 bezüglich des «Schuldetiketts» (i) und der Instrumentalisierung des Einzelnen (ii) in keinem geringeren Mass für den sozialen Schuldbegriff zutreffen, denn selbst die VertreterInnen dieses Schuldverständnis räumen ein, dass zuweilen eine Generalisierung des Schuldmassstabs erforderlich sei, da ansonsten (aufgrund zu vieler exkulpierender Sachverhalte) das Normvertrauen erschüttert würde und die Akzeptanz des Schuldbegriffs in der Gesellschaft infrage gestellt wäre (hiervor Rz. 4). Wenn man solche Generalisierungen bei einem sozialen Schuldbegriff zulässt, wird «Schuld» ebenso zum Etikett. Schuld ist eine normative Zuschreibung und deshalb bei allen Schuldverständnissen zu einem gewissen Masse Etikett.

Wird man sich dieser Tatsache bewusst, zeigt sich auch, 11 dass das Individuum bei jedem Schuldverständnis in einem gewissen Rahmen instrumentalisiert wird. Mit der Instrumentalisierungskritik lässt sich der funktionale Schuldbegriff deshalb nicht erschüttern, da sie für andere Schuldverständnisse ebenso zutrifft. Möchte man also nicht gerade dem Schuldprinzip per se eine Absage erteilen (eine angesichts der Erfahrungen im Massnahmenrecht und der nicht gleich wirksamen Limitierungswirkung des Verhältnismässigkeitsprinzips nicht zu empfehlende Option), ist der partielle Instrumentalisierungsaspekt ein konzeptioneller Kompromiss

26 NIGGLI/MÄDER (Fn.16), S. 310, welche ausführen, dass der Schuldbegriff so manipulierbar werde, weil er nur auf Präventionsbedürfnisse aufbaue.

27 BSK StGB-BOMMER, Vor Art. 19 N 54 ff.; ROXIN/GRECO (Fn. 3), \$19 N35 m.w.H.; kritisch zur präventiven Verankerung des Schuldverständnisses auch HENNING ROSENAU, Rechtliche Grundlagen der psychiatrischen Begutachtung, in: Venzlaff et al. (Hrsg.), Psychiatrische Begutachtung, Ein praktisches Handbuch für Ärzte und Juristen, 7. Aufl., München 2020, S. 85 ff., 89; NIGGLI/MÄDER (Fn.16), S.311; MÜLLER (Fn. 25), S.143f.;

28 MÜLLER(Fn. 25), S.140 ff.; TATJANA HÖRNLE, Straftheorien, 2. Aufl., Tübingen 2017, S. $28 \mathrm{ff}$. 
jedes Schuldbegriffs. Immerhin erinnert die Instrumentalisierungskritik daran, dass die Strafe gegenüber der individuell betroffenen Person zu begründen und rechtfertigen ist. ${ }^{29}$

Sofern man weiter bei einem funktionalen Schuldbegriff den Verlust der strafbarkeitsbegrenzenden Wirkung befürchtet (iii), basiert dies auf seiner unzutreffenden inhaltlichen Konkretisierung. Der funktionale Schuldbegriff knüpft bei richtiger Auslegung - wie von STRENG präzise herausgearbeitet - nicht an eine beliebige Grösse (z.B. ein nicht näher bestimmtes und manipulierbares Normbestätigungsinteresse der Gesellschaft) an, sondern basiert auf der Annahme, dass die gerechte (individuumsbezogene bzw. tatproportionale) Schuldstrafe ${ }^{30}$ zugleich die generalpräventiv wirksamste Reaktion des Staates ist: Insofern sind auch die Befürchtungen zum Verlust der Begrenzungsfunktion nicht gerechtfertigt, da auch auf einem der positiven Generalprävention aufgebauten Schuldverständnis die Limitation der als gerecht eingestuften Strafe besteht. ${ }^{31}$ Dabei muss (iv) jedoch eingestanden werden, dass die plausible Annahme, nur eine gerechte Strafe führe zu Normstabilisierung, bestenfalls approximativ empirisch nachgewiesen werden kann. ${ }^{32}$

29 Vgl. HÖRNLE, Straftheorien (Fn. 28), S. 48f., die entsprechende Begründungsmöglichkeiten zusammenfasst, S. $50 \mathrm{fff}$, und zum Schluss kommt, dass die Bestrafung zu generalpräventiven Zwecken zur Aufrechterhaltung des Gesellschaftssystems fair ist, da die Täterin selber von diesem funktionierenden System mit sich normkonform verhaltenden Personen profitiert hat, vgl.S. 56f.; DIES., Gegenwär tige Strafbegründungstheorien: Die herkömmliche deutsche Diskussion, in: von Hirsch/Neumann/Seelmann (Hrsg.), Strafe - Warum?, Gegenwärtige Strafbegründungen im Lichte von Hegels Straftheorie, Baden-Baden 2011, S. 23, 30; ANDRISSEK (Fn. 25), S.119f.

30 Was gerecht ist, kann bspw. durch einen Rückgriff auf modern verstandene Vergeltungsprinzipien im Sinne der Gerechtigkeitsempfindungen der Bevölkerung erfolgen, was im Endeffekt auf einer empirisch fundierten Theorie einer retributiven Generalprävention hinausliefe. Vgl. hierzu ANDRISSEK (Fn. 25), S. 86, 122, et passim; TONIO WALTER, Grundlagen einer empirisch begründeten Vergeltungstheorie, in: Kaspar/Walter (Hrsg.), Strafen «im Namen des Volkes»?, S. 49 ff.; DERS. Die Vergeltungsidee als Grenze des Strafrechts, JZ 13/2019, S. 649 ff.; TATJANA HÖRNLE, Tatproportionale Strafzumessung, Berlin 1999 S. 94 f.; «funktional-absolut» bei FRANZ STRENG, Strafrechtliche Sanktionen, Die Strafzumessung und ihre Grundlagen, 3. Aufl., Stuttgart 2012, N16; ferner MÜLLER (Fn. 25), S.149.

31 STRENG, Schuld ohne Freiheit? (Fn. 12), S. $286 \mathrm{ff}$. und insb. $292 \mathrm{ff}$.; auch DERS., Strafrechtliche Sanktionen (Fn. 30), N14 ff., 25, 34; KALOUS (Fn. 19), S. 65 ff.; Offen muss an dieser Stelle bleiben, ob das gerechte Strafmass normativ oder empirisch zu ermitteln ist, vgl. HÖRNLE, Tatproportionale Strafzumessung (Fn. 30), S. 94 f.; zumindest WALTER spricht sich überzeugend für eine empirische Fundierung aus, vgl. WALTER, Grundlagen (Fn. 30), S. $49 \mathrm{ff}$.

32 Vgl. HÖRNLE, Tatproportionale Strafzumessung (Fn. 30), S. 90 ff., insb. $93 \mathrm{ff}$. Dass diese Annahme immerhin hochplausibel ist, legt MÜLLER (Fn. 25) mittels Rückgriffs auf die Gerechte-Welt-Forschung überzeugend dar, S. 83 ff., $149 \mathrm{ff}$., und kommt zum Schluss, dass die delinquenzbedingte Bedrohung des Gerechte-Welt-Glaubens dann bestmöglich bewältigt wird, wenn die Bestrafung als proportionale Reaktion auf die Straftat empfunden wird, S.188.
Etwas zugespitzt könnte man sagen, dass die Inhalte ei- 13 nes sozialen Schuldbegriffs sich in ihrem Gehalt in einem richtig konzipierten funktionalen Schuldbegriff wiederfinden. ${ }^{33}$ Daher gilt auch unter einem funktionalen Schuldbegriff, dass Generalisierungen des Schuldmassstabs (vgl. Rz. 4 und 10) nur ganz ausnahmsweise zulässig sind: Nämlich dann, wenn das andere Vorgehen die gesellschaftliche Akzeptanz des Schuldprinzips (und im Reflex das Normvertrauen der Bevölkerung) infrage stellen würde. ${ }^{34}$ Betrachtet man die Gesamtheit dieser Erwägungen, erweisen sich die Einwände gegenüber dem funktionalen Schuldbegriff nicht als tragfähig.

Welche Schlüsse ergeben sich aus dem Gesagten für die 14 Schulddogmatik im Strafrecht? Es sind deren drei: Erstens wird man sich des wackligen Bodens bewusst, auf dem das Schuldstrafrecht und der mit ihm verbundene Anspruch, Gerechtigkeit herbeizuführen, ${ }^{35}$ gebaut ist. Zweitens erweist sich - anders als vom Bundesgericht vertreten - ein funktionales Schuldverständnis als überzeugender als der soziale Schuldbegriff, solange man im Rahmen seiner Anwendung Verantwortung anhand der tradierten (individuumsbezogenen und darum generalpräventiv als gerecht empfundenen!) Kriterien der Schulddogmatik anknüpft. Drittens ist wichtig zu realisieren, dass dieses Modell (so wie von den Autoren definiert) nicht zu anderen Ergebnissen in der Rechtsanwendung führen wird, weil im Rahmen der positiven Generalprävention eben die verantwortungsproportionale und damit als gerecht empfundene Strafe die Normbestätigungsbedürfnisse am besten realisiert. Insofern unterscheidet sich ein funktionaler und sozialer Schuldbegriff weniger mit Blick auf das Ergebnis, sondern mit Blick auf die theoretisch überzeugendere Fundierung des erstgenannten Modells.

\section{Schuldmassstab bei schweren Straftaten (implizite Hemmschwellentheorie)}

Die vorangehenden Ausführungen zum materiellen 15 Schuldbegriff führen uns zu einem zweiten, damit verbundenen Themenkreis. Das Bundesgericht führte aus, anders als die sachverständige Person dürfe das Gericht bei der Beurteilung der Schuldfähigkeit auch die Art der Straftaten berücksichtigen. Für den vorliegenden Fall (versuchte vorsätzliche Tötung) habe die Vorinstanz zu

33 Vgl. auch BSK StGB-BOMMER, Vor Art. 19 N 54.

34 Dies hat Ähnlichkeit mit dem Gedanken bei ANDRISSEK (Fn. 25), S. 121, wonach der Einzelne ausserhalb des Kerns der Menschenwürde als Mittel zum Zweck eingesetzt werden dürfe, wenn die Strafe als «Ultima Ratio der Erhaltung der Gemeinschaft diene».

35 Dazu AnNA ConinX, Von Augen und Zähnen, ContraLegem 2019/2, S. $26 \mathrm{ff}$. 
Recht einen hohen Massstab für die Annahme einer aufgehobenen Schuldfähigkeit verwendet: Bei schweren Straftaten gegen Leib und Leben seien an eine vollständige Aufhebung der Schuldfähigkeit hohe Anforderungen zu stellen. ${ }^{36}$ Aus welchen Gründen diese qualifizierten Anforderungen bestehen sollen, bleibt jedoch unerwähnt.

Eine mögliche Antwort ergibt sich aus dem Blick auf die deutsche Rechtslage. Dort finden sich vergleichbare dogmatische Konstrukte unter dem Begriff «Hemmschwellentheorie»: Das Konzept fusst auf der Annahme einer höheren Hemmschwelle, die Menschen bei der Begehung von schweren Straftaten überwinden müssen. Daraufbasierend wird gefolgert, die tatzeitbezogenen psychischen Defizite der beschuldigten Person müssten bei schweren Delikten besonders ausgeprägt sein, um die Annahme der Schuldunfähigkeit bzw. der verminderten Schuldfähigkeit zu rechtfertigen. ${ }^{37}$

17 Dieses Argument wirkt prima facie plausibel. Es kann kaum bestritten werden, dass Menschen im Allgemeinen höhere Hemmschwellen bei schwersten Delikten (z.B. Tötung eines Menschen) überwinden müssen als bei leichten Straftaten (z.B. Überschreiten der zulässigen Parkzeit). Für diese Hemmschwellen sind Anlage- (z.B. Genetik) und Umweltfaktoren (Sozialisierung, z.B. in Form der Vermittlung des Tötungsverbots etc.) ursächlich. ${ }^{38}$ Nur hilft der Gedanke einer im Normalfall vorhandenen Hemmschwelle bei der Beurteilung der Schuldfähigkeit kaum weiter. Weist die begutachtete Person (tatzeitbezogen) akzentuierte psychopathologische Symptome auf, bewegt man sich definitorisch nicht auf der Ebene des zuvor beschriebenen Hemmschwellen-Normalfalls. Vielmehr ist es bei solchen Personen wahrscheinlich, dass Hemmschwellen aufgrund störungskonnexer Beeinträchtigungen von psychischen Funktionen nicht oder nur unzureichend entwickelt bzw. in ihrer Wirksamkeit reduziert oder eliminiert wurden. ${ }^{39}$ Zurecht hat daher NEDOPIL festgestellt: «Eine empirische Begründung, dass psychisch Gestörte bei schwerwiegenden Verbrechen grössere Hemmungen hatten oder sich von höheren normativen Anforderungen leiten liessen als bei weniger gravierenden Delikten, gibt es nicht.» ${ }^{40}$ Dies überzeugt, denn

36 Urteil des Bundesgerichts 6B_1363/2019 vom 19. November 2020 E.1.7.1.

37 Vgl. dazu FRANZ STRENG, Hemmschwellentheorie, Vorsatz und Schuldfähigkeit, in: Esser et al. (Hrsg.), Festschrift für Hans-Heiner Kühne zum 70. Geburtstag, Heidelberg 2013, S. 47 ff.

38 Eingehend zur Thematik JENNY LEDERER, Hemmschwellen im Strafrecht, Eine übergreifende Untersuchung der Hemmschwellen bei Sexualdelikten und Totschlag, Berlin 2011, S.1ff. und 211 bzgl. der Hemmschwelle des psychisch gesunden Menschen bei Tötungen.

39 LEDERER (Fn. 38), S.19ff. und $135 \mathrm{ff}$.

40 NORBERT NEDOPIL, Forensische Psychiatrie, Psychopathologie zwischen Neurowissenschaft und normativen Zwängen, in: Schöch angesichts der erfolgten Tat (die zur Schuldfähigkeitsfrage ja erst führt) liesse sich im konkreten Fall immer das Argument vorbringen, dass die Missachtung der bei gesunden Personen vorhandenen Hemmschwelle durch das begangene Delikt gerade eine Manifestation von Störungsauswirkungen qualifizierter Schwere ist. ${ }^{41}$

Sofern das Bundesgericht nun ausführt, das Gericht 18 habe bei schwersten Delikten einen besonders strengen Massstab anzulegen, wirft dies Fragen auf. In der Sache lässt sich die bundesgerichtliche Ausführung nur verstehen, wenn man sich an die Ausführungen zum materiellen Schuldbegriff erinnert. Wenn das Hemmschwellenargument im Schuldfähigkeitskontext vorgebracht wird, manifestiert sich in ihm eine vom Bundesgericht als notwendig erachtete Generalisierung des Schuldmassstabs (dazu Rz. 4, 10 und 13f.). ${ }^{42}$

Vereinfacht ausgedrückt dürfte die bundesgerichtliche 19 Ratio lauten, dass die Gesellschaft bei schwersten Delikten eine Schuldunfähigkeit oder verminderte Schuldfähigkeit nur in ganz eng umgrenzten Ausnahmefällen als gerecht erachtet und jedes andere Vorgehen die Akzeptanz des Schuldprinzips infrage stellen würde. Es gibt jedoch gute Gründe, an diesem Schluss zu zweifeln. Es wurde ausgeführt, dass auch unter einem funktionalen Schuldbegriff nur die gerechte (tatproportionale) Strafe eine Normbestätigung sinnvoll herbeiführt. Generalisierungen sind nur ganz ausnahmsweise zulässig: Nämlich dann, wenn es zwingend ist, um die gesellschaftliche Akzeptanz des Schuldprinzips zu erhalten.

Das im vorliegenden Fall eine Erschütterung der Rechts- 20 ordnung bei einem Freispruch wegen Schuldunfähigkeit die Folge gewesen wäre, ist jedoch wenig wahrscheinlich. Die verurteilte Person erlitt infolge Alkoholkonsums eine nicht vorhersehbare Psychose: einen seltenen, schweren psychopathologischen Sonderzustand, in dem Einsicht- und Selbststeuerung mindestens schwer vermindert, wenn nicht sogar aufgehoben waren (vgl. die Ausführungen im Bundesgerichtsentscheid zu den sich widersprechenden Gutachten). In Kenntnis dieser aussergewöhnlichen medizinischen Sachlage hätte es die Gesellschaft und die Akzeptanz des Schuldprinzips wohl kaum nachhaltig erschüttert, wenn ein Freispruch erfolgt wäre. Vielmehr erschiene hier ein Schuldspruch als

(Hrsg.), Strafverteidigung, Revision und die gesamten Strafrechtswissenschaften, Festschrift für Gunter Widmaier zum 70. Geburtstag, Berlin 2008, S. 933.

41 NEDOPIL (Fn. 40), S. 933

42 Vgl. auch STRENG, Hemmschwellentheorie (Fn. 37), S. 57, der hier den Brückenschlag zum sozialen Schuldbegriff und den damit wiedergegebenen normativen Erwägungen der Rechtsprechung als unübersehbar bezeichnet. Vgl. auch «Normativer Filter» bei HÖRNLE, Tatproportionale Strafzumessung (Fn. 30), S. $316 \mathrm{ff}$. 
ungerecht, welcher den gravierenden Auswirkungen des Störungsbilds nicht Rechnung trägt. Daher wäre es bei Fällen dieser Art - der hier vertretenen Meinung nach nicht nötig gewesen, den Schuldmassstab zu generalisieren. Diese Erwägungen sollte in einem weiteren Rahmen auch bei anderen Schwerstdelikten gelten: Primärer Ansatzpunkt sollten die tatzeitbezogenen (störungskonnexen) Auswirkungen der Einsichts- und Steuerungsfähigkeit sein. Insofern steht die Hemmschwellentheorie in einem Spannungsverhältnis mit einem auf das Individuum ausgerichteten Schuldbegriff. ${ }^{43}$

\section{Zulässigkeit von gutachterlichen Empfehlungen zur Schuldfähigkeit}

Im letzten Analyseteil ist die Aufmerksamkeit auf die bundesgerichtlichen Ausführungen zum Sachverständigenbeweis zu richten. Das Bundesgericht führte aus, die sachverständige Person habe im Rahmen der Schuldfähigkeitsbegutachtung die Aufgabe, den biologisch-psychologischen Zustand der tatverdächtigen Person zu beurteilen. Allerdings müsse sie bei dieser Arbeit keine normative Abstinenz walten lassen, sondern sie dürfe ihre Befunde in juristische Begriffe (verminderte Schuldfähigkeit, Schuldunfähigkeit) kleiden. ${ }^{44}$ Die nun zu analysierende Frage lautet, ob sich diese Schlussfolgerungen vor dem Hintergrund des Sachverständigenrechts rechtfertigen.

er hier betrachtete Bundesgerichtsentscheid erfolgt zu einem spannenden Zeitpunkt, da im Jahr 2020 ein neuer Fragenkatalog für forensisch-psychiatrische Gutachten der SSK publiziert wurde. ${ }^{45}$ Während die Fragestellung des bis im Jahr 2020 geltenden Fragenkatalogs für forensisch-psychiatrische Gutachten im Strafrecht direkt auf die Schuldfähigkeit gerichtet war ${ }^{46}$ (z.B. Grad der verminderten Schuldfähigkeit), sieht der neue Fragenkatalog von der Verwendung dieses Begriffs ab. ${ }^{47}$ Der Revisionsvorschlag wurde von URBANIOK/MATHYS/WEDER kritisiert: Die normative Abstinenz der sachverständigen

43 Dies unabhängig davon, ob man einen sozialen oder funktionalen Schuldbegriff zugrunde legt.

44 Urteil des Bundesgerichts 6B_1363/2019 vom 19. November 2020 E.1.6.1.

45 Schweizerische Staatsanwälte-Konferenz SSK, Fragenkatalog für forensisch-psychiatrische Begutachtungen, 3.Juli 2020, ausgearbeitet durch die Arbeitsgruppe Rechtsmedizin und Forensische Psychiatrie der SSK in Zusammenarbeit mit Vertretern der Schweizerischen Gesellschaft für Forensische Psychiatrie SGFP.

46 Vgl. Fragen zu Punkt2 des Anhangs in der Weisung des Kantons gerichts und der Oberstaatsanwaltschaft (Luzern) über psychiatrische und aussagepsychologische Gutachten im Strafverfahren vom 7. Mai 2014.

47 Anzufügen ist, dass es sich auch beim neuen Fragenkatalog nicht um eine vollständige Abkehr vom Stellen von Rechtsfragen handelt, weil immer noch explizit auf Rechtsnormen Bezug genommen wird.
Person bringe keine Qualitätsverbesserung mit sich. Es sei unumgänglich, dass sich die sachverständige Person auch mit Fragen rechtlicher Natur befasse, zumal Tatund Rechtsfragen oft nicht sauber zu trennen seien. Die gutachterliche Stellungnahme zu juristischen Inhalten sei unproblematisch, da das Gericht in seiner normativen Entscheidung freibleibe. Die adäquate Fragestellung an die sachverständige Person laute daher: «Wie ist unter Berücksichtigung der Einsichts- und Steuerungsfähigkeit (vgl. Art.19StGB) aus gutachterlicher Sicht die Schuldfähigkeitfür diezu beurteilenden Straftaten einzuschätzen? Bei eingeschränkter Schuldfähigkeit: In welchem Grad (leicht, mittel, schwer) ist die Schuldfähigkeit aus gutachterlicher Sicht vermindert?». 48

Das Bundesgericht kam in seinem Urteil zum Ergebnis, 23 das in der Sache der Auffassung von URBANIOK/MATHYS/ WEDER entspricht, d.h. auch die sachverständige Person darf sich zur Schuldfähigkeit bzw. deren Verminderung oder Aufhebung äussern. Es überrascht jedoch, dass die Feststellung nicht ausführlicher hergeleitet wurde. In seiner bisherigen Rechtsprechung hatte sich das Bundesgericht in einzelnen Entscheiden dafür ausgesprochen, dass sich die sachverständige Person zur Rechtsfrage, ob eine verminderte Schuldfähigkeit vorliege, nicht zu äussern habe. ${ }^{49} \mathrm{Im}$ ausserstrafrechtlichen Kontext stellte es in einem Leitentscheid sogar fest, dass das Gericht nicht auf eine Expertenaussage abstellen dürfe, wenn mit ihr eine Rechtsfrage beantwortet werde: ${ }^{50}$ Bei der Schuldfähigkeit soll nun anderes gelten. Dafür rekurriert das Bundesgericht auf seine bisherige strafrechtliche Rechtsprechung und die h.L., zu deren Ermittlung auf den Basler Kommentar und die dort von BOMMER ${ }^{51}$ konsultierte Literatur verwiesen wird. ${ }^{52}$ Dieses Vorgehen wirft jedoch Fragen auf: Erstens werden beim Verweis auf die eigene Rechtsprechung just jene Urteile weggelassen, welche die Unzulässigkeit gutachterlicher Stellungnahmen $\mathrm{zu}$ Rechtsfragen stipulierten (vgl. eingangs der vorliegenden

48 FRANK URBANIOK / HANS MATHYS / ULRICH WEDER, Fragenkatalog für psychiatrische Gutachten in Strafverfahren, Konzeption und Zielsetzungen des Fragenkatalogs T18, AJP 2020, S.1573ff., $1576 \mathrm{f}$.

49 Urteil des Bundesgerichts 6B_853/2016 vom 18. Oktober 2017 E.3.4: "Im Hinblick auf das psychiatrische Sachverständigengutachten und dessen Berücksichtigung ist darauf hinzuweisen, dass sich der Gutachter zu der vom Gericht zu beurteilenden Rechtsfrage, ob eineverminderte Schuldfähigkeit im Sinne von Art. 19 Abs. 2 StGB vorliegt, nichtzuäussern hat.»; Urteile des Bundesgerichts 6B_1092/2009 und 6B_67/2010 vom 22.Juni 2010 E.3.1.

50 BGE130 I337 E. 5.4.1: «Dem Sachverständigen sind bloss Sach-, keine Rechtsfragen zu unterbreiten. Die Beantwortung letzterer obliegt zwingend dem Gericht (...). Darausfolgt, dass das Gericht nicht aufeine Expertenaussage abstellen darf, wenn mit dieser eine Rechtsfrage beantwortet wird.»; Urteil des Bundesgerichts 1B_213/2020 vom 4. August 2020 E. 3.2: «Lıexpertise ne doit jamais porter sur une appréciation juridique des faits.»

51 BSK StGB-BOMMER, Art. 20 N33f.

52 Urteil des Bundesgerichts 6B 1363/2019 vom 19. November 2020 E.1.6.1. 
Randziffer). Zweitens wäre u.E. der Kreis der herrschenden Lehre weiter zu ziehen: Zwar trifft es zu, dass ein wesentlicher Teil der materiellstrafrechtlichen Lehre zu Art. 19f. StGB (im Bundesgerichtsurteil via BOMMER zitiert) sich für die Zulässigkeit gutachterlicher Empfehlungen zur Schuldfähigkeit ausspricht oder diese zumindest toleriert. ${ }^{53}$ Es überrascht aber, dass das Bundesgericht mit keinem Wort auf die Diskrepanz zur strafprozessualen Literatur eingeht. Hätte es diesen Schritt unternommen, wäre es zur Feststellung gelangt, dass das strafprozessuale Schrifttum mancherorts das Postulat formuliert, dass der sachverständigen Person nur Sachverhalts- und keine Rechtsfragen zu stellen sind und dass Aussagen der sachverständigen Person zu Rechtsfragen einen Ausstandsgrund nach sich ziehen können. ${ }^{54}$

Hätte man anschliessend die forensisch-psychiatrische Literatur analysiert, wäre man in einem Standardwerk auf das folgende Zitat von DRESSING/FOERSTER gestossen: «Keinesfalls darf sich der Sachverständige zu Formulierungen versteigen wie etwa: «Ich bescheinige erheblich verminderte Schuldfähigkeit, was ein krasser Fehler und ein ebenso krasses Überschreiten der eigenen Kompetenz wäre.»55

Vor diesem Hintergrund wäre das Bundesgericht verpflichtet gewesen, sorgfältig zu begründen, warum es im Rahmen der Schuldfähigkeit ausnahmsweise (in Abweichung der Best Practice des Sachverständigenrechts nach Art. 182 ff. StPO, Teilen der forensisch-psychiatrischen

53 So BSK StGB-BOMmer, Art. 20 N 34 m.w.H.; a.A. aber GIAN EGE, Der Affekt im schweizerischen Strafrecht, Diss. Zürich 2017, S.334 ff. m.w.H., insb. Fn. 2055; SCHMID (Fn. 5), S. 449; für Deutschland bspw. STRENG, StrafrechtlicheSanktionen(Fn. 30), N 961 und dort Fn.3433 m.w.H.

54 NIKLAUS SCHMID / DANIEL JOSITSCH, Schweizerische Strafprozessordnung (StPO), Praxiskommentar, 3. Aufl., Zürich 2018, Art.184 N 8 (gemäss diesen Autoren sind Fragen nach der Schuldfähigkeit unzulässig); MARIANNE HEER, in: Niggli/Heer/Wiprächtiger (Hrsg.), Basler Kommentar zur Schweizerischen Strafprozessordnung (StPO)/Jugendstrafprozessordnung (JStPO), 2. Aufl., Basel 2014, Art. 182 N 4 sowie Art. 184 N16 (wo ausgeführt wird, es könne sich aufdrängen, «zum Voraus die Abgrenzung der Kompetenzen zwischen dem Auftraggeber und der sachverständigen Person zu klären, etwa die sachverständige Person zum Verzicht auf die Beantwortung von Rechtsfragen anzuhalten»); NIKLAUS OBERHOLZER, Grundzüge des Strafprozessrechts, 4. Aufl., Bern 2020, N1015 mit Verweis auf Urteil des Bundesgerichts 8C_448/2015 vom 17. Dezember 2015 E. 4-5, E. 4.2; etwas permissiver ANDREAS DONATSCH, Art. $184 \mathrm{~N} 20$, in: Do natsch et al. (Hrsg.), Kommentar zur Schweizerischen Strafprozessordnung (StPO), 3. Aufl., Zürich 2020, wonach in der Praxis das Postulat, dass Sachverständige sich der Beantwortung von Rechtsfragen enthalten, in der Schuldfähigkeitspraxis nicht konsequent ein gehalten wird und dafür hält, dass dies solange toleriert werden könne, wie das Gericht die Rechtsfrage unabhängig von der sachverständigen Person entscheide und begründe. Insofern in Reibung mit Art.183 N16, wo die Annahme eines Ausstandsgrunds thematisiert wird, wenn sich die sachverständige Person zu Rechtsfragen äussert.

55 HARALD DRESSING / KLAUS FOERSTER, Aufgaben und Stellung des psychiatrischen Sachverständigen, in: Venzlaff et al. (Hrsg.), Psych iatrische Begutachtung, Ein praktisches Handbuch für Ärzte und Juristen, 7. Aufl., München 2020, S.7.
Literatur und partiell sogar seiner eigenen Rechtsprechung) legitim ist, dass sich die sachverständige Person zu einer Rechtsfrage (Schuldfähigkeit) äussert. Wäre es dieser Begründungspflicht nachgekommen, hätte man gemerkt, dass gute Gründe für das Postulat der normativen Abstinenz der sachverständigen Person sprechen. Eine gutachterliche Stellungnahme zur Rechtsfrage der Schuldfähigkeit liesse sich nämlich nur dann rechtfertigen, wenn der auf Sachverhaltsebene erhobene medizinische Befund bzw. die quantifizierten Beeinträchtigungen der Einsichts-/Steuerungsfähigkeit mit Schuldfähigkeit, verminderter Schuldfähigkeit oder Schuldunfähigkeit gleichgesetzt werden könnte. Dies ist aber nicht der Fall (vgl. II.1): Zwar bestehen Zusammenhänge dahingehend, dass die normative Verantwortungszuschreibung im Rahmen des funktionalen Schuldbegriffs wesentlich von psychischen Funktionsbeeinträchtigungen abhängt, die von der sachverständigen Person erhoben und quantifiziert werden (darin liegt die von URBANIOK/MATHYS/ WEDER zurecht angesprochene Überlappung von Tatund Rechtsfrage). Die Übersetzung des medizinischen Befunds in eine normative Verantwortungszuschreibung enthält aber, wie unter II. aufgezeigt, weitere Gesichtspunkte und ist insofern eine Rechtsfrage. ${ }^{56}$

Anerkennt man diese Differenz von medizinischem Tat- 26 sachenbefund und rechtlicher Verantwortungszuschreibung, könnte auf pragmatischer Ebene argumentiert werden, dass eine gutachterliche Empfehlung zur Schuldfähigkeit solange unproblematisch sei, wie sich das Gericht nicht an diese Einschätzung gebunden sehe. ${ }^{57}$ Wenn letzteres flächendeckend gewährleistet wäre, bestünde in der Tat kein Problem. Der Haken an dieser Auffassung ist aber, dass die dafür vorausgesetzten Begebenheiten (richterliches Autonomieverständnis, Wissen um materiellen Schuldbegriff etc.) in der Praxis nicht immer vorliegen. Vielmehr bergen gutachterliche Schuldfähigkeitsempfehlungen Gefahren: Gesteht man der sachverständigen Person diese Kompetenz zu, besteht das Risiko, dass die gutachterliche Empfehlung zur Schuldfähigkeit durch die Tatsache, dass sie von einer psychiatrisch sachverständigen Person stammt, (zu Unrecht!) einen wissenschaftlichen Anstrich erhält, obwohl es sich angesichts des unter II. beschriebenen Schuldbegriffs um eine gesellschaftlich-normative Zuschreibung handelt. Zurecht wird von KRÖBER (einem forensischen Psychiater)

56 ELMAR HABERMAYER / JOSEF SACHS, Aufgaben und Rolle der forensischen Psychiatrie, in: Jusletter vom 27. April 2015, Rz. 4 mit dem berechtigten Hinweis, dass keine Parallelität zwischen medizinischem Befund und juristischer Schuldfähigkeit bestehe.

57 Dies war bis vor kurzem auch die Meinung eines Co-Autors, von der er nach reiflicher Überlegung mit der nachfolgenden Begründung abweicht. Vgl. THIERRY URWYLER, Das Teilnahmerecht der Verteidigung am Explorationsgespräch des psychiatrischen Sachverständigen mit der beschuldigten Person im Lichte der EMRK, Mit Fokus auf die Gutachten zur Schuldfähigkeit und Massnahmenindikation, Diss. Luzern 2019, N130 f. 
betont, dass «Schuldfähigkeit bei manchen den falschen Eindruck erweckt, es handele sich um einen psychobiologischen Sachverhalt» und nicht um das, was es ist: eine normative Zuschreibung. 58

Für strafbehördliche Entscheidungsträgerinnen, die sich dieses komplexen Verhältnisses von Tat- und Rechtsfrage nicht bewusst sind, ist bei gutachterlichen Schuldfähigkeitsempfehlungen nicht mehr klar, was Teil der medizinischen Sachverhaltseinschätzung ist, welche das Gericht vorbehaltlich triftiger Gründe im Rahmen der Sachverhaltsfeststellung bindet, und was eine Aussage zu Rechtsfragen darstellt, die vom Gericht zwar zur Kenntnis genommen werden kann, aber von der ohne Bindungswirkung abgewichen werden darf. Hinzu kommt: Selbst wenn sich Strafbehörden der Kompetenzenordnung zwischen Sachverständigen und Gericht noch bewusst wären, könnten sie geneigt sein, es sich im Sinne einer falsch verstandenen Prozessökonomie einfach zu machen, indem sie die gutachterliche Empfehlung unbesehen übernehmen und sie nicht mehr kritisch in den rechtlichen Referenzrahmen einbinden.59 In einem solchen Fall wäre die nicht delegierbare Aufgabe des Gerichts zur Rechtsanwendung (iura novit curia) in unzulässiger Weise auf die sachverständige Person übertragen. ${ }^{60}$

Schliesslich stellt sich die Frage, warum man der sach verständigen Person das exklusive Privileg zur Abgabe einer Schuldfähigkeitsempfehlung einräumt und dasselbe Recht weiteren Personen im Verfahren (z.B. Zeuginnen, Auskunftspersonen usw.) nicht zugesteht. Letztere wären angesichts des gesellschaftlich-normativen Charakters des Schuldbegriffs gleich gut (oder, bei einem pessimistischeren Verständnis der Erkenntnismöglichkeiten von Menschen, gleich schlecht) qualifiziert zu beurteilen, ob bzw. in welchem Rahmen angesichts der auf Sachverhaltsebene vorliegenden (störungskonnexen) psychischen Funktionsbeeinträchtigungen der Einsichtsund Steuerungsfähigkeit unter juristisch-normativen Gesichtspunkten ein Schuldvorwurf an diese Person gerichtet werden kann. Wenn aber die sachverständige Person hinsichtlich des rechtlichen Schuldbegriffs keine überlegenen Fachkenntnisse aufweist, ist ihre Meinung dazu nicht einzuholen, denn ihre strafprozessuale Funktion beschränkt sich darauf, im Rahmen der Sachverhaltsfeststellung Fachwissen in das Verfahren einzubringen (in casu: störungskonnexe Einschränkungen hinsichtlich

\footnotetext{
58 HANS-LUDWIG KRÖBER, Konzepte und Implikationen der verminderten Schuldfähigkeit, FPPK 2020, S. 384.; auch ANDRISSEK (Fn. 25) S.130: «Schuld ist das, was eine Gesellschaft als Schuld ansieht».

59 Dabei handelt es sich nicht um eine rein theoretische Befürchtung Vielmehr zeugen diverse von den Autoren gesichtete Urteile/Gutachten, dass Gerichte die gutachterliche Schuldfähigkeitsempfehlung teilweise unverändert übernehmen und nicht mehr eigenständig würdigen.

60 So auch SCHMID (Fn. 5), S. 449.
}

tatbezogener Einsicht und Steuerung), und nicht, ihre allgemein-gesellschaftliche Auffassung von individueller Verantwortung zu äussern, wie es jeder andere Bürger oder jede andere Bürgerin auch könnte.

Vor diesem Hintergrund ist es sinnvoll, wenn sich die 29 sachverständige Person auf die Diagnose einer tatzeitbezogenen Störung und den daraus ggfs. folgenden Beschreibung der störungsbezogenen Funktionsbeeinträchtigungen - d.h. den Sachverhalt! - beschränkt. ${ }^{61}$ Bis das Bundesgericht (und die Begutachtungspraxis) dem hier formulierten Gedanken Rechnung trägt, gilt es zu betonen, dass Gerichte nicht an gutachterliche Empfehlungen zur Schuldfähigkeit gebunden sind, sondern unter normativen Gesichtspunkten davon abweichen können. ${ }^{62}$

\section{Zusammenfassung}

Was ist der Ertrag dieses Beitrags? Die vorangehenden 30 Ausführungen ergeben erstens, dass ein soziales Schuldverständnis dogmatisch "auf halbem Wege stehen»63 bleibt. Nur ein funktionales Schuldverständnis lässt sich widerspruchsfrei herleiten, wobei auch auf dessen Boden die individuumsbezogene Zuschreibung von Verantwortlichkeit das Leitprinzip bleibt. Insofernändern sich mit seiner Anwendung nicht die Ergebnisse, sondern das theoretische Fundament.

Zweitens konnte gezeigt werden, dass bei schwersten 31 Straftaten nur dann mit Hemmschwellenargumenten operiert werden sollte, wenn ansonsten die Akzeptanz des Schuldprinzips selbst gefährdet wäre, was nach hier vertretener Meinung im vorliegenden Verfahren nicht der Fall war.

Drittens und abschliessend wurde dargelegt, dass sich 32 die sachverständige Person im Rahmen der forensischpsychiatrischen Begutachtung nicht zur normativen (Rechts-)Frage der Schuldfähigkeit äussern sollte. Damit verlässt sie den Bereich ihrer eigenen Expertise (Sachverhalt) und greift in den Kernkompetenzbereich des Gerichts (Rechtsanwendung) ein.

61 Die hier vertretene Lösung soll nicht darüber hinwegtäuschen, dass selbst dann die gutachterlichen Quantifizierungen der störungsbezogenen Funktionsbeeinträchtigungen - die auch mit dem neuen Fragenkatalog anfallen - eine gewisse Suggestivwirkung auf die strafbehördliche Rechtsanwendung entfalten werden. Dennoch ist es für die Gerichte nicht mehr so leicht bzw. angenehm, schlicht das gutachterliche Verdikt zu übernehmen.

62 Dies wird in der Praxis zuweilen getan: vgl. HENNING HACHTEL / LORENZ AENIS / DERYA SAHIN / MARC GRAF, Der Einfluss des Sachverständigengutachtens auf das Urteil aus psychiatrischer und juristischer Sicht, SZK 2014, S. 3 ff.

63 Vgl. im analogen Kontext in Deutschland: STRENG, Schuld ohne Freiheit? (Fn. 12), S. 276, 296. 